\title{
REVIEW
}
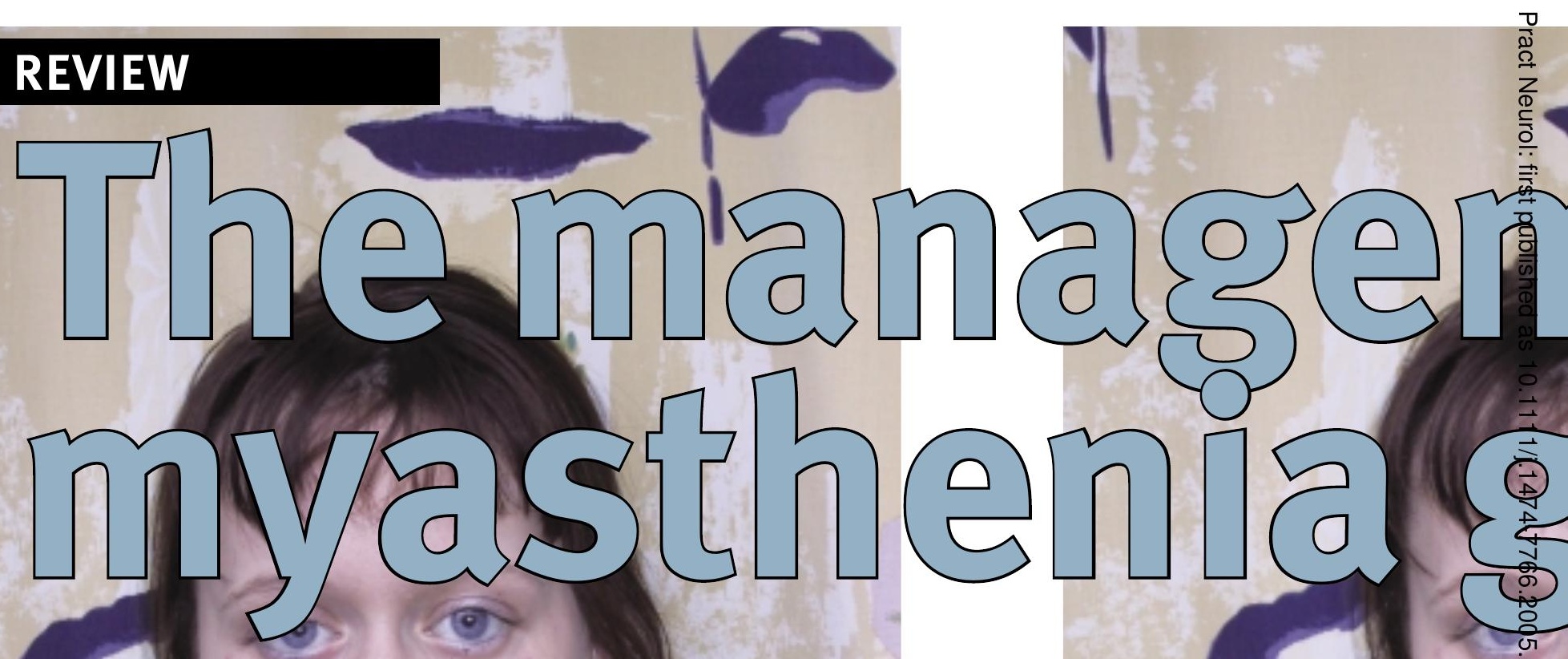

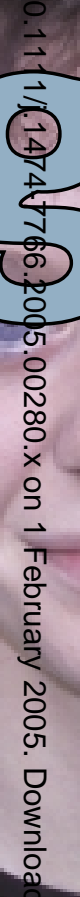

David Hilton-J ones and J ackie Palace

Consultant Neurologists, Myasthenia Gravis Association Myasthenia Centre,

Radc liffe Infirmary, Woodstock Road, Oxford OX2 6HE;

E-mail: david.hilton-jones@clinical-neurology.oxford.ac.uk

Practical Neurology, 2005, 5, 18-27 


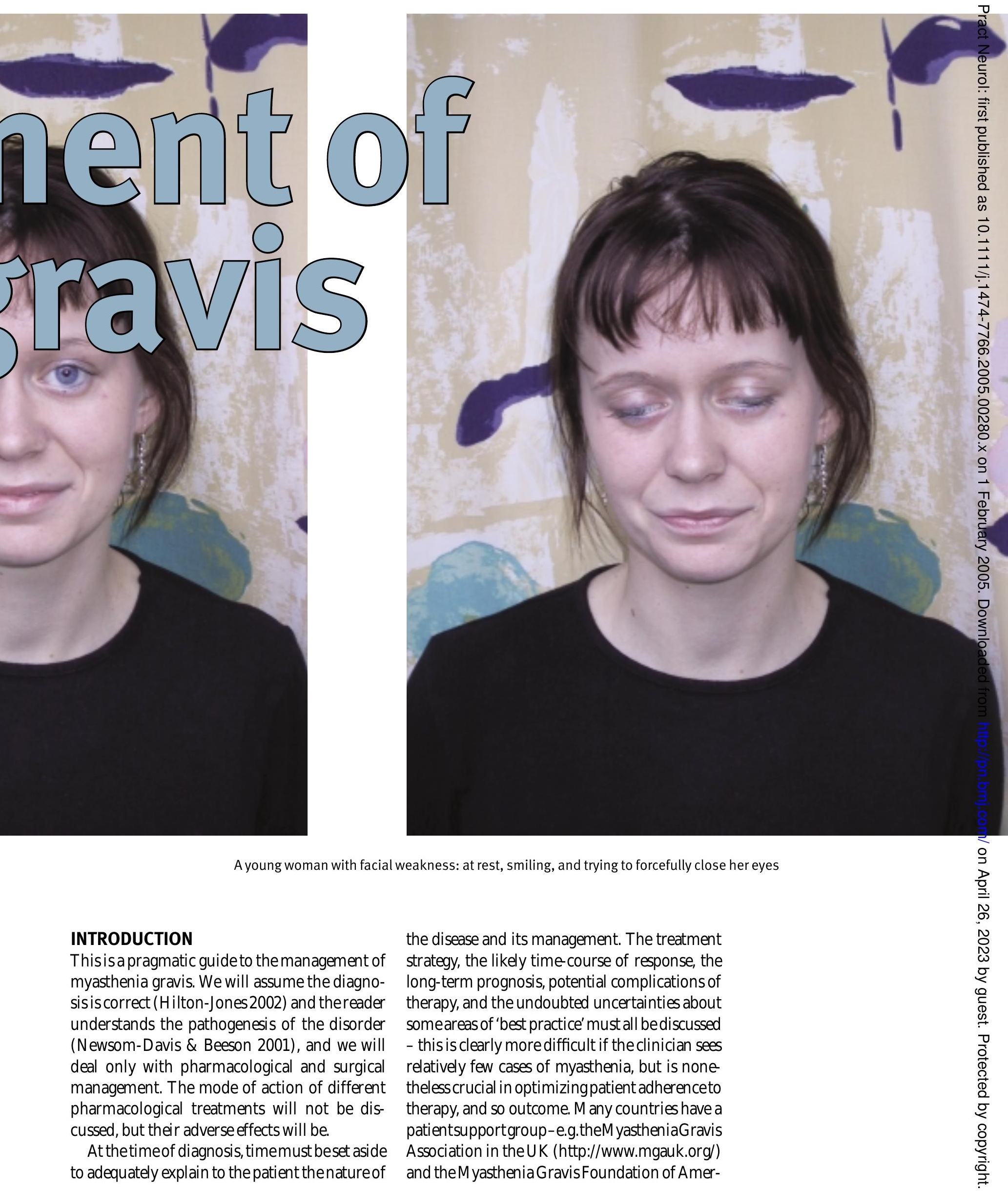


ica in the USA (http://www.myasthenia.org/) - which provides literature (typically less sensational than some of the information on the internet); this is a useful starting point for patients and their primary care physician on what is often going to bea very long journey.

\section{PRINCIPLES OF MANAGEMENT}

Themanagement of myastheniagravisconfined to the extraocular muscles (ocular myasthenia) differs somewhat from that of generalized myasthenia (and later thetwo conditions will be discussed separately). However, theprinciples of management aresimilar, and for both there are three fundamental approaches:

- symptomatic treatment;

- immunomodulatory therapy;

- immunosuppressant therapy.

\section{Symptomatic treatment}

\section{Anticholinesterases}

M ost patientswill respond, often quitedramatically, to an anticholinesterase, but in only a very small proportion will thisbeadequatelong-term therapy. Itmustbeemphasized to thepatient that oncethediseasehas been brought under control by other treatments, then it should be possible to withdraw theanticholinesterase. N ot making this clear at the outset partly explains why some patients have such difficulty withdrawing their anticholinesterase at a later date when they are in remission. One can understand their reluctance to withdraw a drug that, sometimes after months of uncertainty about diagnosis often tinged with suggestions of non-organicity, had dramatically improved their weakness. The drug may also have a non-specific stimulatory effect that patientsparticularly noticeif thedrug is suddenly withdrawn, so gradual withdrawal over several weeks is the best strategy.

There is no reason to use any anticholinesterase other than pyridostigmine. Although a're tard' formulation is now widely available, and has been much talked about on the web, there have been no trials demonstrating its superiority over the standard preparation. There is also some anxiety, based on theory rather than experience, that the longer duration of action and thus accumulation may precipitate cholinergic crisis. If the patient is struggling to adequately control symptoms with pyridostigmine, then it is probably timeto moveon to other therapeutic strategies.
Pyridostigmine should be introduced slowly (Box 1) to reduce the risk of adverse effects, which are essentially restricted to smooth muscle stimulation causing abdominal cramping, increased flatus, diarrhoea and, less frequently, urinary frequency and incontinence. If abdominal symptoms develop, they can be treated by propantheline- $15 \mathrm{mgtds}$, or $15 \mathrm{mg}$ beforeeach dose of pyridostigmine, or once daily, whatever is required for symptomatic control. For those who have difficulty swallowing, the pyridostigminetablets can becrushed.

Cholinergic crisisshould belargely confined to the history books - it was caused by excessive doses of anticholinesterases, which were not that uncommon when no other treatment options were available. It is still very occasionally seen as a result of physician or patient ignorance. If the maximum dose of pyridostigmine is confined to six tablets ( $=360 \mathrm{mg}$ ) daily in adults, our experience is that cholinergic crisis never occurs.

\section{Extra-ocular muscle surgery}

Rarely, ptosis may be the only significant persisting symptom of myasthenia gravis, in which case ptosis-correction surgery may be a more attractive proposition than immunosuppressant therapy if pyridostigmine alone isn't adequate. In our experience, few patients tolerate ptosis-barsfitted to their glasses. Si milarly, some patients may be left with isolated diplopia due to fixed weakness of one or more extra-ocular muscles and the use of a prism or surgical correction may be appropriate (and preferable to the introduction of a steroid sparing immunosuppressant drug in those with pure ocular myasthenia).

BOX 1 ANTICHOLINESTERASE (PYRIDOSTIGMINE) THERAPY

- Pyridostigminetablets are $60 \mathrm{mg}$.

- They are scored and so can be divided into quarters (15 mg).

- Start with 15 mg qds.

- Increase (if necessary) after 2 days to 30 mg qds.

- Increase (if necessary) after 2 days to 60 mg qds.

- Maximum dose 360 mg daily

- usually 90 mg qds

- but some patients may benefit from $60 \mathrm{mg} \times 6$ daily.

- Add propantheline if gastrointestinal adverse effects 


\section{Any drug which}

\section{had been}

\section{introduced}

shortly before a

relapse should

be considered a

possible cause.

In practice, $\beta$ -

blockers are

probably the

most common

culprit.

\section{Immunomodulatory therapy}

We use this term to describe those treatments (intravenous immunoglobulin, plasma exchange, and thymectomy) that alter thebody's immune system without frankly suppressing it. Intravenous immunoglobulin (IVIg) or plasma exchange (PE) are used as quick acting but temporary measures to improve function whilst waiting for other therapies to take effect, whereas thymectomy is intended to offer long-term benefit. IV I gand PE may lead to improved function within days of starting treatment, but their ben eficial effect lasts no longer than 6- 8 weeks.

IVIg and PE

Both of these (expensive) treatments are widely used despite a paucity of evidence of benefit. It is perhaps easiest to summarize the situation by quoting the recent Cochranereview:

'One randomised controlled trial did not show a significant difference between intravenous immunoglobulin and plasma exchange for treatment of severe exacerbations of myasthenia gravis. There is no evidence from randomised controlled trials to determine whether intravenous immunoglobulin for moderate or severe myasthenia gravis improves functional outcome or has a sparing effect on steroid dosage, nor is there sufficient evidence to favour intravenous immunoglobulin over steroids in moderate exacerbations. Further randomised controlled trials are needed' (Gajdos et al. 2003).

Other smaller trials and anecdotal reports suggest there is no significant difference between theapparent benefits of IVIg and PE, so it is appropriate to consider them together.

Of course, the lack of trials does not necessarily mean that the treatments are ineffective and indeed few experienced observers doubt their efficacy. What is unanswered is whether they are superior (in terms of clinical benefit and cost) to other treatment options, such as simply increasing the dose of steroids in a myasthenic relapse. Our practice, paralleled by many others, is to use IVIg in preference to $P E$ except when an individual patient has been intolerant of IVIg, or has previously responded better to PE than IVIg. IVIg is simpler to administer and can be used in the presence of systemic infection, the latter point being particularly important as sepsis is often a trigger for myasthenic crisis.

The main indications for IVIg/PE are:

- newly diagnosed patient with substantial weakness requiring a quick therapeutic effect;

- myasthenic relapse/crisis in a previously diagnosed patient.

In the newly diagnosed patient who is clearly going to need morethan pyridostigmine(i.e.inadequatebenefit after increasing up to $6 \times 60 \mathrm{mg}$ tablets a day in 2-3 weeks), the main treatment options (see below) are thymectomy or immunosuppression. IVIg may improve the patient prior to thymectomy - and reducetheir perioperativerisk. Patientsstarting prednisolone, even when introduced gradually, may show an initial deterioration of their myasthenic symptoms in the first few weeks of therapy. Furthermore, the full response to prednisolone may take many months. IVIg is given in the hope of giving early improvement in function, carrying the patient through until theprednisolonestarts to become effective, and lessening thelikelihood of any initial prednisolone-induced deterioration. Whilst this practiceis now quitewidely established, the need for a formal trial is readily apparent. IVIg is expensive, not only in terms of the product itself, but also the need to admit the patient to hospital.

Deterioration in a patient whose myasthenia was previously controlled (a relapse, or if very marked referred to as myasthenic crisis) may be:

- spontaneous (which is not that uncommon and reflects thespontaneousfluctuation seen in many autoimmune disorders);

- induced by 'stress' (e.g. infection, surgery, emotional issues, hormonal factors);

- caused by reduction or withdrawal of previously effectivetherapy;

- precipitated by recent introduction of a drug that interfereswith neuromuscular transmission. Although many drugs are known to do this (Newsom-Davis\& Beeson 2001), listsare not exhaustive and any drug which had been introduced shortly before a relapse should be considered a possible cause. In practice, $\beta$-blockers are probably the most common culprit.

Any underlying cause must be treated or removed (e.g. infection) and immunosup- 
pressive therapy may need to be increased, particularly if the relapse was due to reduction of therapy. Within thelimitations already noted, IVIg is widely used as a short-term measure to improve function whilst waiting for any infection, for example, to settle, and any increased immunosuppressive therapy to become effective.

The main practical problem with IVIg is aseptic meningitis. If patients develop typical symptoms we do not perform a lumbar puncture, but just stop the IVIg and treat with anal gesics and the problem usually settles within 3-5 days. This reaction may be product-specific and wehave sometimes, with the patient's consent, successfully used an alternative product without reaction on subsequent occasions. Prior to first use of IVIg many believe the patient should haveablood test to excludelgA deficiency (patients with IgA deficiency produce antibodies against the IgA in the IVIg). In all cases the patient must be counselled about the potential hazardsfrom ablood-donor-derived product

\section{Thymectomy}

Few doubt that thymectomy works for some people - but which ones? Despite having been used widely for over 50 years the definitive trial is still now only in the planning stages - that it is going to happen at all is largely the result of a recent review (Gronseth \& Barohn 2000). On the available, not very good, evidence, including personal experience, our current practice is asfollows.

Most patients with thymoma identified on chest M RI or CT undergo thymectomy, all of ours being performed via the sternal split approach. An exception is the very elderly patient in whom serial scanning to monitor progress may be more appropriate. The patients are advised that their myasthenia is very unlikely to respond to thymectomy, but surgery is required to treat the thymoma, because there is significant morbidity associated with local and intrathoracic spread of the tumour. As an aside, it must be noted that there are recent data concerning tumour stage and histological subtypeand theneed for adjuvant therapies (radiotherapy and chemotherapy) that any department conducting thymectomy for tumour must be aware of (Chen et al. 2002). M RI and CT are probably about equal in detecting thymoma and the choice is up to local availability and radiologist preference. 0 ccasionally there may be uncertainty as to whether or not a thymoma is present, in which case thescan should be repeated after a year. Scans are not used to diagnose hyperplasia, which is a pathological diagnosis (Fig. 1).

For patients without thymoma who fulfil the following criteria, we advise that thymectomy givesa $25 \%$ chanceof remission, a $50 \%$ chanceof improvement but still having to take long-term medication, and a $25 \%$ chance of no benefit:

- generalized (rather than purely ocular) myasthenia gravis;

- age under 45 years;

- anti-acetylcholine receptor antibody positive

- within 1-2 years of onset.

Benefit may sometimes be apparent within weeks of thymectomy, but can be delayed for up to two years. Werecognizethat it isvery difficult to determine whether any benefit accrues from the surgery, or from other changes in therapy, or indeed because of spontaneous fluctuation of the disease, which is well documented, hence our support for the proposed international trial of thymectomy

We do not advise thymectomy for older patients, in whom thethymusistypically atrophic, or for patients who are anti-acetylcholine receptor antibody negative (no antibody negative cases have been reported with true thymic hyperplasia), although we are aware that some recognized authorities do. There is

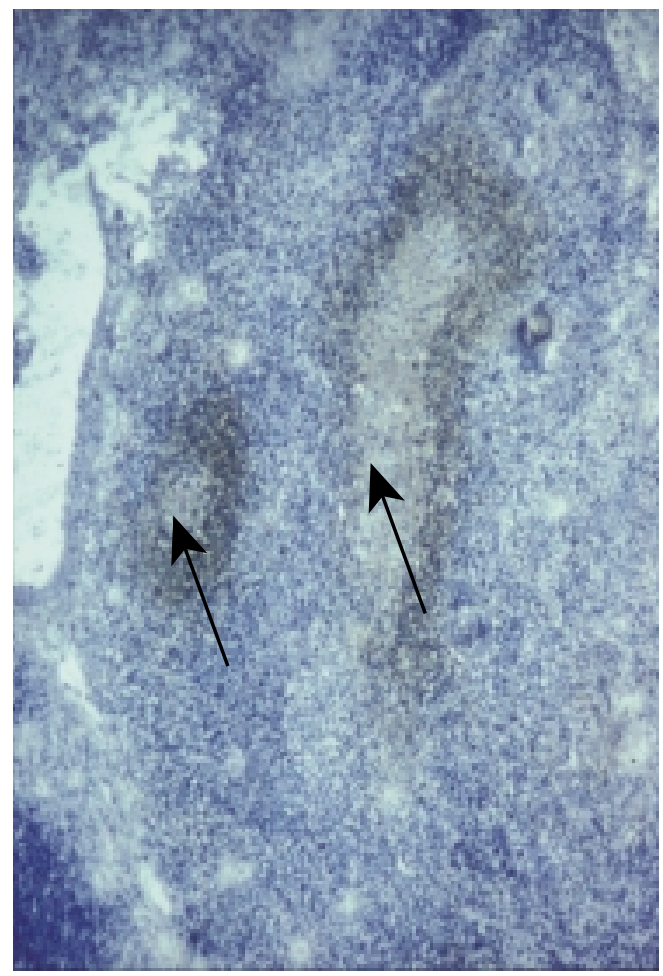

Figure 1 Thymic hyperplasia: CD22 anti-B-cell antibody stained section of a hyperplastic thymus showing two germinal centres (arrows) surrounded by Blymphocytes. 
as yet inadequate experience in patients who are anti-M uSK antibody positive to make useful comment.

\section{Immunosuppressant therapy}

Prednisoloneisthemainstay of immunosuppressant therapy. Other immunosuppressant drugs (e.g. azathioprine, methotrexate, ciclosporin, mycophenolatemofetil) aregenerally considered 'second-line' or 'steroid-sparing', butoccasionally they areused alone(e.g. when a patient is intolerant of, or refuses, steroids). With one exception - azathioprine - there is a dearth of randomised trials, and practice is based on personal experience, and prejudice.

Pregnancy merits comment. There is no evidence that either prednisolone or azathioprine are teratogenic, but there have been reports of prematurebirth and low birth-weight following exposureto azathioprine, particularly in combination with steroids. All the other immunosuppressant drugs are relatively contraindicated in pregnancy, mainly because of lack of evidence rather than certain evidence of problems. M ethotrexate is also contraindicated in males who wish to becomefathers because it can lower the sperm count; whilst thereis no certain evidence of teratogenesis it is recommended that males stop taking thedrug at least threemonths before attempted conception. But it must be emphasized that the contraindication in pregnancy is relative - the risks need to be balanced against the benefits. Additionally, maternal transfer of antibodiesismorelikely to occur in inadequate- ly treated myasthenia. The key is informed discussion.

\section{Prednisolone}

The symptoms of myasthenia gravis may undoubtedly deteriorate following introduction of prednisolone but how much this is due to the drug, how much to the mode of introduction (e.g. starting at a high dose rather than gradually increasing), and how much to unrelated causes such asthenatural history of thediseaseafter first presentation, is unclear. Few people advocatethe useof high-doseoral or intravenousmethylprednisolone as used in multiple sclerosis (e.g. $2.5 \mathrm{~g}$ over 2-5 days) - a few small studies have given contradictory results with either evidenceof benefit or deterioration (there may bescopefor further study, perhaps combined with IVIg, of the potential benefit for earlier remission).

When surveying theM yastheniaCentresplanning to takepart in theinternational thymectomy trial, about $80 \%$ used alternate- day prednisolone. Theevidencein adults that adverse effects are fewer than when using daily steroids is dubious but that isstill our practice. Early fluctuation (i.e. worseon thenon-steroid day) seemsto beagood prognostic feature of steroid responsiveness and usually settles with timeasstrength improves. An occasional exception to persevering with an alternateday regimeisfor thosepatientswith diabetes whose diabetic control proves difficult because of daily fluctuations. We always introduce prednisolone gradually (Box 2). Steroids take weeks to monthsto work and thereisno good reason to

\section{BOX 2 IMMUNOSUPPRESSION WITH PREDNISOLONE AND AZATHIOPRINE FOR GENERALIZED MYASTHENIA GRAVIS}

In general, the patient should be admitted for initiation of therapy. Even with gradual introduction of prednisolonethere can berapid deterioration with risk of bulbar and respiratory problems. Theintroduction of immunosuppression may run concurrently with IVIg/PE (seetext).

\section{- Prednisolone}

- Usealternate day dosage from theoutset.

- Initial dose $10 \mathrm{mg}$.

- Increaseby 10 mg every dose until target dose reached or, rarely, symptoms and signs resolveat a lower dose.

- Target dose $=1.5 \mathrm{mg} / \mathrm{kg}$ body weight on alternate days, or $100 \mathrm{mg}$ on alternate days, whichever is the lower.

\section{- Azathioprine}

- Target dose $=2.5 \mathrm{mg} / \mathrm{kg}$ body weight/day.

- Use a bd regime because there is a lower risk of gastro-intestinal adverse effects than once daily, e.g. nausea and diarrhoea.

- Start on 25 mg once daily.

- Increase by 25 mg daily until on target dose.

- Check full blood count and liver function every third day until up to target dose, then weekly for onemonth, then monthly for three months, and then three monthly. 
think that initial high-dosetherapy is going to be the better option - and there is no doubt that it often leads to initial deterioration. So introduce steroids gradually in all patients, and use IVIg or $P E$ to get an early response in particularly severe cases.

Patients must be given detailed counselling (including written information) about the potential adverseeffectsand complication sof steroids. These include increased risk of infections, osteoporosis, hypertension, diabetes, cataracts, and peptic ulceration. Often underemphasized problems, which frequently complicatetherapy, include mood disturbance (depression, mood swings, psychosis) and insomnia. M any patients are particularly concerned by the prospect of weight gain. In our experience, steroid-myopathy does not develop in patientson alternateday prednisolone, even when used at high dosage. Additional drug therapy to prevent osteoporosis and peptic ulceration are summarized later. Standard 'Steroid Information Cards' should of coursebeissued.

\section{Azathioprine}

It has been shown that combining azathioprine with prednisolone lowers the eventual steroid requirement and so reduces steroid-related adverse effects such as weight gain (Palace et al. 1998). H owever, azathioprine is slow to act and benefit may not appear for 12 months. Thedrug should therefore be used for at least 24 months at appropriate dose before deeming it ineffective. Our practice is to introduce azathioprine with prednisolone at the outset of treatment in patients with generalized myasthenia (Box 2).

About $10 \%$ of patients are intolerant of the drug with symptoms developing shortly after its introduction (notably nausea, vomiting or diarrhoea). Potentially more severe problems are liver and bone marrow dysfunction and, whatever other measures are taken, blood tests (full blood count and liver function) must be performed every three days or so until it is clear thereareno adversereactions. Once patientsare on the full dose of azathioprine, the blood tests should be weekly for one month, then monthly for three months, then three-monthly. Macrocytosis, lymphopenia, and mild elevation of bilirubin and transaminases are normal reactions to the drug but may alarm the primary care physician organizing the blood tests unless informed.

Azathioprine is metabolized by the enzyme thiopurine methyltransferase (TPMT). Allelic polymorphisms of the TPM T gene determine enzyme activity. Those patients who lack enzyme activity (about 1 in 300) are bound to develop myelosuppression, which is potentially fatal, with conventional doses of azathioprine. If the assay is available then it is sensible to use it. However, even those with 'normal' enzyme activity can develop myelosuppression and hepatitis and so the golden rule must still be to regularly monitor the full blood count and liver function from the outset. Those with high levels of enzyme activity may need higher than standard doses of the drug to achieve the desired effect. One approach is to use a standard target dose (e.g. $2.5 \mathrm{mg} / \mathrm{kg}$ body weight/day) and, where there is an inadequate therapeutic response, and no significant lymphopenia or macrocytosis, to increase the dose to $3 \mathrm{mg} / \mathrm{kg}$ body weight/day.

\section{Other immunosuppressant drugs}

Numerous immunosuppressant drugs other than azathioprine have been used and there is no reason to doubt that they can be effective (Box 3). However, none has yet been subjected to an adequate randomised trial so the benefit to harm ratio is unknown. They probably work more rapidly than azathioprine. We will confine comment to those that arefairly widely used - the order issimply al phabetical. All carry the risk of myelosuppression and hepatitis and regular blood monitoring is required.

Ciclosporin is widely used in transplant medicinesothereisconsiderableexperienceavailable. At the dosesnormally used for treating myasthenia, serum level estimation is not required. $\mathrm{Hy}$ pertension, impaired renal function (watch the serum creatinine) and hypercholesterolaemia are relatively frequent complications. Hirsutes, tremor, peripheral paraesthesiaeand crampsare common adverse effects.

Methotrexate is probably the most widely used of all second-line immunosuppressant

\section{BOX $_{3}$ OTHER IMMUNOSUPPRESSANTS}

Target doses are given. Full blood count and liver function must be monitored, and in the case of ciclosporin also creatinine.

- Ciclosporin $2.5 \mathrm{mg} / \mathrm{kg}$ body weight/day in two divided doses.

- Methotrexate (in an adult) 7.5-20 mg once weekly (+ folate).

- Mycophenolatemofetil $1 \mathrm{gbd}$.
Once the

patient is in

remission the

pyridostigmine should be withdrawn gradually over 2-4 weeks. If symptoms and signs return, the patient is not in remission. 
drugs (at least in rheumatological and dermatological practice), which hastheadvantagethat primary care physicians are familiar with it and havewell-established monitoring programmes. It is generally well-tolerated but the potential for pulmonary involvement must be noted (reported predominantly in patientswith rheumatoid arthritis on relatively high doses), and for the interaction with aspirin and non-steroidal anti-inflammatory drugs (both of which may increase serum methotrexatelevels and thusincrease the risk of toxicity). Concomitant use of folic acid reduces adverse effects.

$M$ ycophenolatemofetil has recently received a lot of publicity in the myasthenia patient literature. A randomised trial isplanned to start soon. This drug has the advantage of a simple fixed dosage regime ( $1 \mathrm{~g}$ twice daily). There is some evidence, which requires confirmation, that it may act more quickly than the other drugs discussed.

\section{A PRACTICAL APPROACH}

\section{Initiation of treatment}

Figures 2 and 3, together with Boxes 1-4, outlinethemanagement of ocular and generalized myasthenia gravis, respectively. The management of myasthenia in patients with thymoma is the same, but as noted above the thymoma should be removed in most patients. In all patients having thymectomy, whether for a thymoma or as management of the myasthenia, peri- and postoperative complications will be less if the myasthenia is well-controlled at the time of surgery - IVIg or plasma exchange about a month before surgery may be appropriate (see above).

There are conflicting opinions, within and between different countries, concerning osteoporosis prophylaxis when long-term relatively high-dose steroid therapy is being used. Some advocate bone density scanning and treating only thosewith an abnormality, others notethat steroid-induced osteoporosis can develop within months of starting treatment and therefore bisphosphonates should be introduced from the outset. Some advocate different approaches to high and low risk groups of patients. When available, it would seem most appropriate to follow existing locally agreed guidelines. Box 5 shows our approach. We continue a bisphosphonateand calcium/vitamin D throughout the duration of steroid therapy.
BOX 4 PREDNISOLONE FOR OCULAR MYASTHENIA GRAVIS

It can beintroduced moreslowly than in generalized myasthenia, as an outpatient

- Usean alternate day dose from the outset

- Maximum dose typically $0.75 \mathrm{mg} / \mathrm{kg}$ body weight/alternate days, or $60 \mathrm{mg}$ on alternate days, whichever is lower.

- Initially 5 mgon alternateday for threedosesthen 10 mgon alternatedays for three doses.

- Continueincreasing at same rate either until symptoms resolve or target dose is reached.

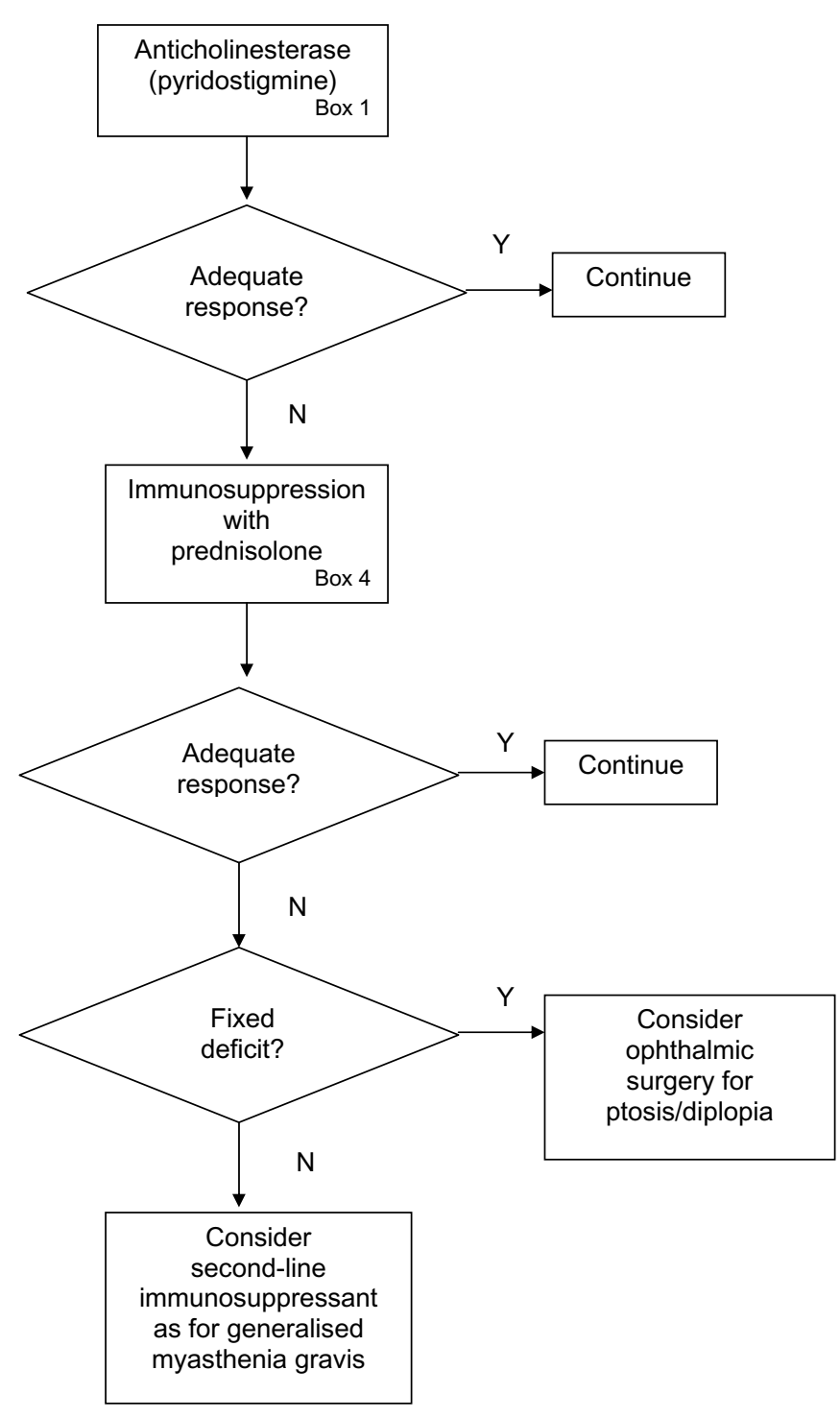

Figure 2 Management of ocular myasthenia gravis. 


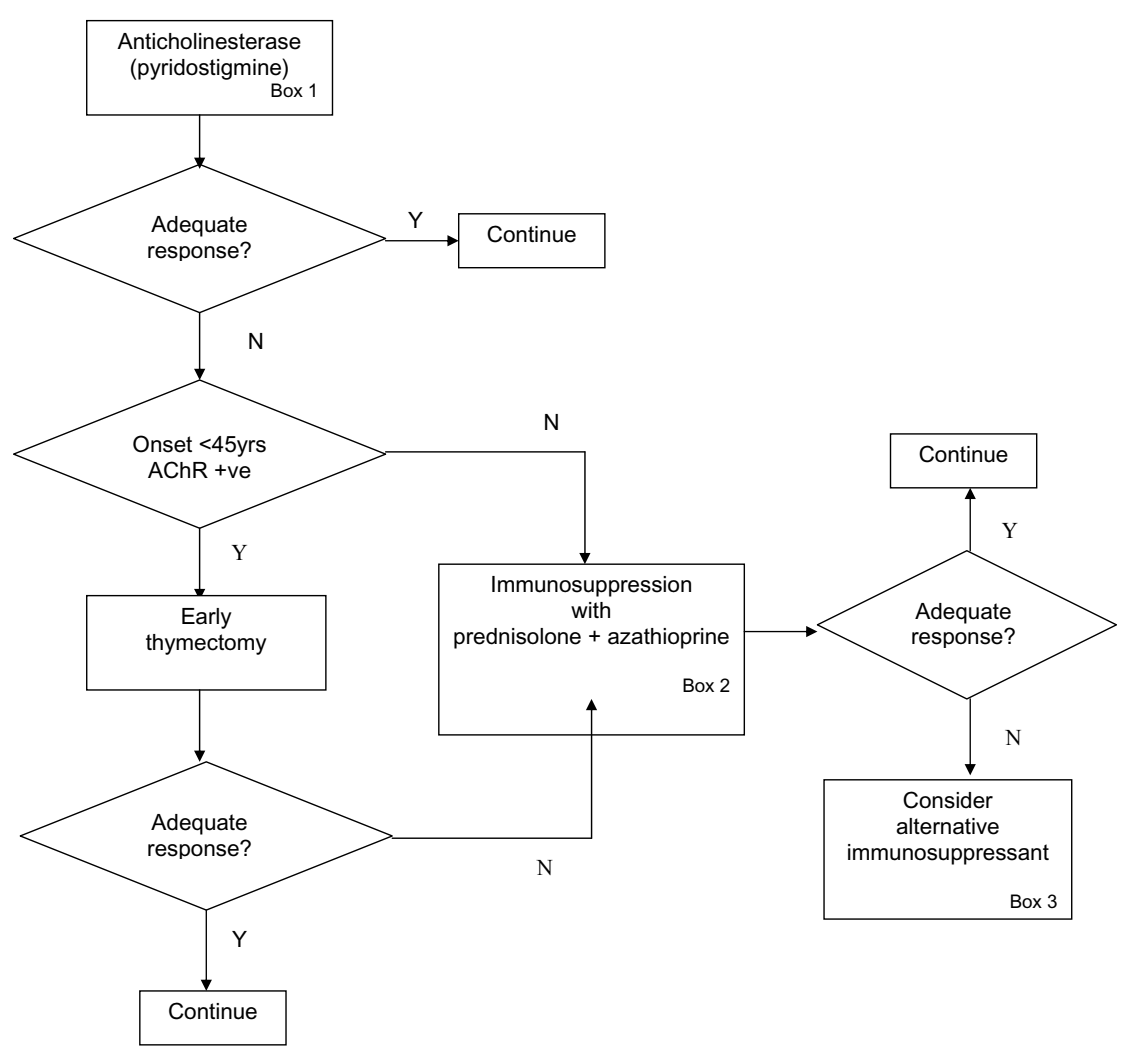

Figure 3 Management of generalized myasthenia gravis ACR, anti-acetylcholine receptor antibody.

\section{BOX 5 OSTEOPOROSIS MANAGEMENT AND STOMACH}

\section{PROTECTION}

- Alendronate $70 \mathrm{mg}$ weekly from start of steroid therapy (the choice of bisphosphonate may be dictated by local policy)

- Calcium/vitamin D combined tablet (many preparations are available). The vitamin D dose islow (typically $800 \mu \mathrm{g}$ daily) and should not induce hypercalcaemia (but pre-existing hypercalcaemia should be excluded). Again, local policy may dictate the preferred preparation.

- Bone density (DEXA) scan as soon as possible after initiation of steroids - repeated after 2 yearsor earlier if indicated.

- General advice about exercise/smoking/alcohol/etc.

- A proton pump inhibitor (e.g. lansoprazole) should be given from the outset

\section{After remission}

Ocular myasthenia often responds within 12 months of initiating treatment and thedosage of prednisolonerequired to achieveremission is usually substantially lower than in generalized myasthenia. A common error is to fail to appreciate that generalized myasthenia may takea long time to enter remission, and anxiety about the relatively high dose of prednisolone may lead to it being reduced too soon.

Once the patient is in remission (which in practicemeansminimal residual symptomsand signs) thepyridostigmine should bewithdrawn gradually over 2-4 weeks. If symptoms and signs return, the patient is not in remission.

The prednisolone should then be reduced gradually, trying to determine the minimum dose required to keep thedisease under control. In generalized myasthenia gravis a reasonable approach is:

- reduceby $10 \mathrm{mg} / \mathrm{month}$ until down to $40 \mathrm{mg}$ on alternate days;

- then reduce by $5 \mathrm{mg} / \mathrm{month}$ until down to $20 \mathrm{mg}$ on alternate days;

- then reduce by $1 \mathrm{mg} / \mathrm{month}$.

A slower rate of prednisolone reduction when nearing the previously noted critical dosage 
may be appropriate in patients who have previously had a relapse on steroid withdrawal. In some patients it may be possible to withdraw the prednisolone completely and retain control with azathioprine alone. Patients with ocular myasthenia can often be maintained on a very low dose of prednisolone.

\section{Managing a relapse}

Relapse is most often due to reduction of immunosuppression or intercurrent illness, particularly infection. In patients with poorly controlled disease the introduction of certain drugs may exacerbate the myasthenia, e.g. $\beta$ blockers or certain antibiotics. Any precipitating cause should be identified and dealt with. $M$ ild relapses may respond adequately to raising the dose of prednisolone, possibly with a short course of pyridostigmine to give symptomatic benefit. For a moresevererelapseIVI gor plasma exchange may be helpful by giving short-term benefit whilst waiting for the increased dose of prednisolone to become effective. Particularly in asevererelapsecloseattention mustbepaid to ventilatory function with intensive monitoring of the forced vital capacity.

\section{THE FUTURE}

The current immunosuppressant approach to treating myasthenia gravis, and indeed many other autoimmunediseases, is non-specific and reminiscent of a blunderbuss. Current research isaimed at a better understanding of theafferent and efferent limbs of the immune process with thehope, and indeed expectation, that it will one day be possible to target only the pathway involved in causingthemyasthenia, without damaging other parts of the immune system. Until then further research is needed to determine the most effective way to use currently available treatments such as thymectomy, and immunosuppressant drugs other than azathioprine.

\section{ACKNOWLEDGEMENTS}

This article was refereed by Richard D aven port, Edinburgh

\section{REFERENCES}

Chen G, Marx A, Wen-Hu C et al. (2002) New WHO histologic classification predicts prognosis of thymic epithelial tumors: a clinicopathologic study of 200 thymoma cases from China. Cancer, 95, 420-9.

GajdosP, Cheveret S\& Toyka K (2003) Intravenous immunoglogulin for myasthenia gravis. CochraneDatabase Syst Review, 2: CD 002277.

Gronseth GS \& Barohn RJ (2000) Practice parameter: thymectomy for autoimmune myasthenia gravis (an evidence-based review): report of the Quality StandardsSubcommittee of theAmerican Academy of Neurology. Neurology, 55, 7-15.

Hilton-JonesD (2002) How to do it: Diagnose myasthenia gravis. Practical Neurology, 2, 173-7.

Newsom-DavisD \& Beeson D (2001) Myasthenia gravis and myasthenic syndromes. autoimmune and genetic disorders. In: D isorders of Voluntary M uscle. (eds Karpati G, Hilton-Jones D \& Griggs R), 7th edn, pp. 660-75. CambridgeUniversity Press, Cambridge.

Palace], Newsom-Davis] \& LeckyBA (1998) randomised double-blind trial of prednisolonealone or with azathioprine in myasthenia gravis. Myasthenia Gravis Study Group. Neurology, 50, 1778-83.

\section{PRACTICE POINTS}

- In over $90 \%$ of patientswith general ized myastheniait should bepossibleto get them back to normal or near-normal strength, and for them to lead a normal life-style

- Less than $5 \%$ will havemajor persisting problems.

- But in most patients requiring long-term immunosuppression someadverse effects areinevitable, although usually tolerable.

- Don't use excessive doses of pyridostigmine(i.e. > 360 mg daily)

- If pyridostigmine does not give adequate control of symptoms other forms of treatment (e.g. thymectomy or immunosuppression) should beconsidered.

- Getting myasthenia under control takes time (many months in most cases) - neither the patient nor the doctor should becomeimpatient.

- Time spent in discussion with the patient early-on will pay dividends in thelong term.

- Myasthenia is not a disease to be managed by a different doctor every timethe patient comes to clinic - continuity of care is essential.

- Therearestill many unanswered questions to do with theoptimum use of thymectomy and immunosuppressant drugs. 